\title{
L'enseignement supérieur au Liban : un paysage complexe
}

Yvette Gharib et Nisrine Hamdan Saadé

\section{OpenEdition}

1 Journals

Édition électronique

URL : https://journals.openedition.org/ries/6029

DOI : 10.4000/ries.6029

ISSN : 2261-4265

Éditeur

France Education international

\section{Édition imprimée}

Date de publication : 1 décembre 2017

Pagination : 18-23

ISBN : 978-2-85420-616-6

ISSN : $1254-4590$

Référence électronique

Yvette Gharib et Nisrine Hamdan Saadé, «L'enseignement supérieur au Liban : un paysage complexe ", Revue internationale d'éducation de Sèvres [En ligne], 76 | décembre 2017, mis en ligne le 01 décembre 2019, consulté le 25 juin 2021. URL : http://journals.openedition.org/ries/6029 ; DOI : https://doi.org/10.4000/ries.6029

Ce document a été généré automatiquement le 25 juin 2021.

(c) Tous droits réservés 


\title{
L'enseignement supérieur au Liban : un paysage complexe
}

\author{
Yvette Gharib et Nisrine Hamdan Saadé
}

1 Depuis la fin de la guerre civile, et parallèlement aux programmes de reconstruction du pays, le système éducatif du Liban a énormément progressé, en termes de développement et d'amélioration de sa qualité. Les programmes n'ayant pas été modifiés depuis les années 1970, un plan de restructuration du système éducatif a été conçu en 1994 et mis en application en 1997, qui visait une réforme globale de l'enseignement général et technique.

2 Le système scolaire aspire à l'équité et à l'égalité des chances en matière d'éducation, et la scolarisation est bien assurée par la coexistence du public et du privé, malgré un décalage important entre les deux secteurs en termes de coûts et de qualité. Ce décalage subsiste en dépit de la réforme et de la rénovation du système engagées sur tout le territoire libanais. Selon une enquête menée en 2004, les ménages libanais consacrent plus de $10 \%$ de leur budget aux dépenses d'éducation, dont 3,5\% pour l'enseignement supérieur (Nahas, 2009). C'est le secteur privé qui a ainsi connu une expansion significative, au point de dominer tout le paysage éducatif libanais.

3 Cet article s'intéresse au système d'enseignement supérieur libanais, et cherche à mettre en évidence les facteurs qui expliquent la disparité existant entre le secteur privé et le secteur public.

\section{État des lieux de l'enseignement supérieur libanais}

4 À la différence des pays de la région MENA, l'enseignement supérieur libanais se caractérise dès son origine, au XIXe siècle, par la domination du secteur privé, par des cursus bilingues français et anglais et surtout par l'empreinte religieuse. Même les premiers réseaux scolaires du pays ont été fondés par des missions religieuses, à partir du XVI ${ }^{\mathrm{e}}$ siècle. 
5 L'American University of Beirut (AUB), fondée par les missionnaires protestants en 1866, l'Université Saint Joseph de Beyrouth (USJ), fondée par l'Ordre des pères jésuites en 1875, dominent la carte universitaire libanaise depuis la seconde moitié du XIX ${ }^{e}$ siècle. L'Institut supérieur La Sagesse existe également depuis 1875, l'Université libanaise américaine (LAU) fut fondée en 1924 par des missionnaires protestants, alors qu'elle était depuis 1885 un collège pour filles et dans les années 1930, l'Université Saint Esprit-Kaslik (1938) et l'Université du Moyen Orient (1939) furent créées. Ces universités sont toujours parmi les plus réputées du pays. De plus, trois universités libanaises figurent dans le classement QS World University Ranking 2018 des 500 meilleures universités mondiales en matière d'enseignement, de recherche, d'employabilité, de réputation internationale ${ }^{1}$. Ces mêmes universités ont été classées parmi les vingt meilleures universités du monde arabe, selon le QS University Ranking Arab Region en 2017.

6 Quant à l'enseignement supérieur public, ce n'est qu'en 1951 que l'Université libanaise (UL), unique université publique jusqu'à nos jours, fut fondée dans le but d'assurer la formation des enseignants et instituteurs, dans son école normale supérieure, avant de se développer durant les années 1950 et d'ouvrir progressivement diverses facultés (en 1959 : faculté des sciences sociales, faculté de droit et faculté des sciences; en 1965 : institut des beaux-arts). À l'époque, le Liban représentait un centre intellectuel qui absorbait des flux significatifs d'étudiants de la région, les pays arabes voisins et ceux $\mathrm{du}$ Golfe n'ayant pas encore instauré leurs propres institutions d'enseignement supérieur (Nahas, 2009).

7 À partir des années 1960 et après le premier texte régulateur de 1961, d'autres universités privées sont fondées, comme l'Université arabe de Beyrouth (1961), l'Université Notre-Dame (1987) au Mont Liban, l'Université Balamand (1988) au nord du pays, qui commencent à s'approprier une bonne partie des effectifs des jeunes étudiants, dans un contexte de guerre où la mobilité géographique des étudiants est paralysée. En parallèle, plusieurs facultés de l'Université libanaise, après le déclenchement de la guerre civile en 1975, installent des branches dans diverses régions du pays, qui ne tardent pas à devenir des unités autonomes ayant chacune leur propre fonctionnement (on dénombrait 60 branches en 2015). À la fin de la guerre civile libanaise en 1990, et parallèlement à la politique néolibérale de l'État, l'enseignement supérieur au Liban commence à connaître des modifications structurelles majeures, surtout en raison de l'entrée en scène d'une série de petites universités et d'institutions privées à coûts réduits et à but plutôt lucratif (23 nouveaux établissements d'enseignement supérieur sont créés entre 1995 et 2001).

Actuellement, sur les 44 universités et instituts de l'enseignement supérieur, l'UL possède les effectifs les plus élevés, malgré une baisse significative due à l'émergence de ces petites universités (67 309 étudiants ou $34,6 \%$ du total des étudiants pour l'année 2015, contre $49,5 \%$ en 2004 et $60 \%$ en 2000) ${ }^{2}$.

9 Un pays comme le Liban, qui vit au bord du chaos et dans une région tourmentée par les conflits, se trouve placé devant une série de défis à relever en ce qui concerne son enseignement supérieur. 


\section{Défis et enjeux}

10 L'évolution de l'enseignement supérieur nécessite d'analyser plusieurs facteurs aux niveaux académique, socio-économique et politique.

11 Au niveau académique, la majorité des universités francophones ont adopté le système européen selon le processus de Bologne, sur la base du système ECTS (European Credits Transfer System), avec les trois niveaux de formation (licence, master, doctorat). Pour les universités qui adhèrent au modèle anglo-saxon, il s'agit également d'un système de crédits et de trois cycles d'études (Bachelor, Master, PhD).

12 Suivant les tendances internationales, la majorité des universités se sont lancées récemment dans les procédures d'assurance qualité et d'accréditation. Cet effort fourni pour relever le défi lié à la recherche de la qualité, dans un contexte agité et complexe, se fait aux dépens d'une véritable harmonisation entre les différentes universités, malgré l'existence de divers projets interuniversitaires tels que les projets européens Tempus. Une concurrence à deux niveaux apparaît nettement, d'une part entre les universités privées elles-mêmes et d'autre part entre le secteur privé et le secteur public. Plusieurs universités offrent les mêmes filières de spécialisation sans se coordonner, si bien que chacune a son propre référentiel de compétence relatif à la formation et au profil de sortie de l'étudiant. Selon la réforme de 1997 et malgré des avancées en matière de restructuration des programmes et des curricula, de méthodes d'évaluation et de mise en œuvre de l'approche par compétences, de grandes difficultés apparaissent également en ce qui concerne la formulation des nouveaux programmes, surtout lorsque celle-ci diffère selon l'école des experts convoqués de l'extérieur. Selon le Bulletin statistique du CRDP pour l'année 2004-2005, l'Université libanaise assurait 105 spécialisations théoriques et professionnelles, alors que les institutions universitaires privées en proposaient 160 . De même, il arrive souvent que des colloques traitant de thématiques identiques soient organisés par plusieurs universités, par exemple en ce qui concerne les relations université/entreprise.

13 Ce manque d'harmonisation entre les universités creuse un fossé entre les acteurs de l'enseignement supérieur. Pourtant, la réforme de 1997 avait prévu d'établir des réseaux de communication académique et des bases de données communes entre les établissements du supérieur et les administrations, mais rien n'a encore été fait.

$14 \mathrm{Au}$ niveau socio-économique, malgré tous les efforts consentis pour la restructuration du système éducatif, il existe encore d'énormes lacunes en ce qui concerne la capacité des cursus et programmes à accompagner l'évolution des conjonctures économiques et sociales, en lien avec le marché du travail, afin de faciliter l'insertion professionnelle des étudiants. Malgré la présence de stages dans le parcours des étudiants, des difficultés majeures subsistent en matière d'insertion professionnelle après le diplôme. Les compétences directement utilisables sur le marché du travail ne sont pas toutes intégrées dans les formations de l'enseignement supérieur. Au niveau de la collaboration entre les entreprises et les institutions du supérieur, et malgré les divers efforts entrepris, les résultats restent mineurs. Il n'existe toujours pas de système de formation en alternance université-entreprise dans le supérieur libanais.

$15 \mathrm{Au}$ niveau politique, les ingérences politiques et sectaires dans les affaires de l'UL freinent le développement de l'institution et violent son intégrité, pour des intérêts personnels et électoraux. À titre d'exemple, l'équilibre confessionnel doit être préservé 
pour toute nomination de doyen ou titularisation de professeurs. Pour l'entretien et la réhabilitation des bâtiments de l'UL, la décision reste politique.

Bien que des lois régissent le système éducatif libanais, certaines sont difficilement applicables par les universités. Ainsi, l'obligation d'intégration scolaire est affirmée par une loi (220/2000) en vue d'assurer le droit à l'éducation des personnes à besoins particuliers. Cependant, seul un petit nombre d'universités offrent des formations en enseignement spécialisé (orthopédagogie, orthophonie, psychomotricité, psychopédagogie). Le nombre de diplômés dans ce domaine est toujours insuffisant, ce qui entrave l'application parfaite de la loi 220. D'autres dispositions légales n'ont pas été mises en application, comme le décret $n^{\circ} 31$ du Gouvernement libanais, daté du $16 / 8 / 2000$, portant sur le remplacement du brevet professionnel par un système de formation d'ouvriers compétents, si bien que les seuls textes disponibles et suivis jusqu'ici remontent aux années $1960^{3}$.

17 De plus, l'octroi de licences ne tient pas compte d'une répartition géographique équitable des institutions du supérieur selon une vision soucieuse d'assurer l'accès à toutes les spécialisations. Pourquoi un étudiant d'un village du sud du pays serait-il contraint de venir habiter dans la capitale, s'il souhaite se spécialiser en sciences économiques?

18 Par ailleurs, des lacunes au niveau juridique sont observées quant à la mise en place de textes de lois et de règlements pour répondre aux besoins du marché du travail. Selon un rapport (2006) de l'Association libanaise des sciences de l'éducation ${ }^{4}$, "l'enseignement supérieur souffre de sérieux problèmes tel qu'un système et législation archaïques définissant le cadre des deux secteurs public et privé de cet enseignement». Des licences ont été octroyées à 25 nouveaux établissements d'enseignement supérieur privés au début des années d'après-guerre (1990-2001), sans aucune étude de besoins ou de stratégie nationale préalables. Selon Daccache (2012), « cette poussée universitaire eut lieu, dans un contexte très libéral, sans qu'il y ait une loi-cadre et une administration capable de contrôle et de vérification des programmes enseignés et de la qualité de la formation». Cette extension de l'enseignement supérieur n'était pas nécessairement liée aux besoins de développement du secteur ni à l'évolution des domaines de spécialisation (El Amine et al., 1999). Par contre, plus d'autonomie a été accordée aux établissements privés sans réglementation, responsabilisation institutionnelle ou demande de reddition de comptes. À titre d'exemple, mentionnons qu'un ministre de l'éducation nationale s'est octroyé à luimême une licence pour fonder une université qui s'est rapidement étendue sur le territoire libanais, sans que ceci ne soit perçu par le gouvernement comme un conflit d'intérêts.

19 Cette inadéquation des formations dispensées débouche sur une crise de surplus confirmée par plusieurs indicateurs : $81 \%$ des jeunes de 21 à 25 ans étaient des diplômés en 2007 , contre $66,2 \%$ en 2001 ; le taux de chômage des diplômés est en hausse (11,1 \% en 2014 contre un chômage moyen de 9,2 \% en 2007 (Issa, 2014) ; 20000 jeunes émigrent chaque année et près de 55 \% des diplômés libanais entre 1995 et 2005 vivent actuellement à l'étranger, une perte significative de capital humain. 


\section{Perceptions du terrain}

20 Comment les universités libanaises envisagent-elles de relever les défis actuels de l'enseignement supérieur? Peut-on parler d'absence de coopération entre les institutions du supérieur dans les deux secteurs? Le manque de vision et de planification stratégique des autorités de l'enseignement supérieur est-il à l'origine de la rupture avec la réalité du terrain? Afin d'apporter des éléments de réponse à ces questions, nous avons eu recours à des entretiens menés avec cinq enseignants, un vice-recteur et deux chefs de département, selon un guide d'entretien semi-directif.

21 L'analyse thématique des réponses collectées a mis en évidence une série de termes à connotation négative: "absence d'harmonisation, faible communication interuniversitaire, programmes inadaptés, rigidité de certaines matières, lois non appliquées ", etc. Par contre, d'autres résultats ont dévoilé plusieurs initiatives lancées par les universités des deux secteurs, pour faire face aux défis d'ordre académique essentiellement, plutôt que d'ordre socio-économique et politique.

22 Nous constatons que les acteurs du terrain sont conscients d'une réalité marquée par l'absence d'une stratégie de coopération entre les différentes universités et formulent le souhait de changer cette situation insatisfaisante: "On croit, on espère évoluer, souhait de changer $»$.

23 Ces résultats confirment l'écart qui existe entre le secteur privé et public, surtout en ce qui concerne la concurrence entre les universités, marquée par une absence de coopération et de vision commune pour une éducation de qualité. La disparité entre les deux secteurs se situe également au niveau des conditions matérielles et de la qualité des ressources assurées. Ceci rejoint les résultats d'une étude antérieure concernant l'UL, qui souligne des «lacunes importantes au niveau de la diversification des méthodes, de la disponibilité des ressources et des supports pédagogiques, et du suivi personnalisé de l'étudiant » (Abdul-Reda, 2009).

24 Sans être généralisables, ces résultats reflètent une certaine réalité du terrain marquée par une volonté de vouloir faire progresser l'enseignement supérieur. Les acteurs de terrain se disent tiraillés au quotidien et voient leur potentiel inhibé, aliéné par des contraintes plus politiques et économiques que proprement académiques. Ainsi, l'un des enquêtés relate avec amertume et honte le fait qu'un ancien ministre a fait attribuer à son fils une licence pour fonder une nouvelle université, ensuite vendue à un investisseur.

Malgré ce cas de figure, la situation de l'enseignement supérieur au Liban reste porteuse d'optimisme et de promesses, comme l'a indiqué le recteur de l'USJ dans son allocution d'ouverture de l'année universitaire 2017-2018: « Malgré l'instabilité sociale, sécuritaire et politique qui marque la vie de notre région et de notre pays, nous ne pouvons que compter sur notre clairvoyance, notre esprit de communauté USJ et notre ferme volonté de continuer à être au service de la formation d'une jeunesse compétente et engagée ».

Par ailleurs, de nombreuses associations (ALSE $\left.{ }^{5}, \mathrm{ADMEE}^{6}, \mathrm{LAAS}^{7} . ..\right)$, émanant d'initiatives individuelles, regroupent des enseignants chercheurs de différentes universités désireux de faire évoluer l'enseignement supérieur au Liban, contribuant ainsi à concrétiser le potentiel des acteurs. Les universités ont réagi à leur tour en 
encourageant la recherche dans différents domaines, en matière de formation des enseignants et de contribution au savoir scientifique.

De son côté, l'État a réactivé certaines dispositions prévues par la loi, telles que la constitution d'un comité de onze experts, dont la mission est de vérifier la validation et la légalité de la création de tous les établissements d'enseignement supérieur. D'autre part, l'État développe la coopération internationale, au travers de la signature de plusieurs protocoles avec différents pays européens. Selon Jammal (2017), au niveau local et régional, un projet d'échange d'experts en évaluation et procédures d'assurance qualité a été mis récemment en place; de même, le renforcement de la coopération avec toutes les parties prenantes et le développement d'un cadre national de qualification est prévu et encouragé pour relever les défis. Cette collaboration à l'international se traduit également par des programmes de mobilité universitaire qui concernent les enseignants, les étudiants et les personnels d'administration.

La disparité qui caractérise l'enseignement supérieur libanais existe aussi bien entre les secteurs public et privé qu'entre les universités privées elles-mêmes et elle est surtout déterminée par des facteurs socio-économiques et politiques. Mais le secteur éducatif libanais a toujours su remonter la pente (rétablissement rapide après-guerre, conflits politiques internes) grâce aux diverses initiatives privées et publiques et à son ouverture au monde.

\section{BIBLIOGRAPHIE}

ABDUL-REDA A. S. (2009) : «Le Liban », in B. Labaki, Enseignement supérieur et marché du travail dans le monde arabe, Beyrouth : Presses de l'Ifpo, p. 217-245.

DACCACHE S. (2012) : « Le système éducatif libanais : un regard d'ensemble des réalités, des problèmes et des défis ", intervention au colloque Fondation Oasis : Maison de la Montagne, Beyrouth.

EL-AMINE A. et al. (1999) : L'Université libanaise et sa réforme, Beyrouth.

JAMMAL A. (2017): « Panorama de l'enseignement supérieur », Sixth Mena Regional Tertiary Education Conference « Towards Competitiveness and Equity in Tertiary Education in the MENA Region Collaboration for Good Governance, Sustainable Financing and Internationalization », 15-16 juin, Marseille.

NAHAS C. (2009) »: Financing and Political Economy of Higher Education in Lebanon, Beirut: Economic Research Forum.

\section{NOTES}

1. AUB, USJ et LAU.

2. Source: CRDP : Centre de recherche et de développement pédagogiques, Bilan statistique 2015-2016. 
3. La Stratégie nationale de l'éducation et de l'enseignement au Liban, rapport de l'Association libanaise des sciences de l'éducation (ALSE), décembre 2006.

4. Ibid.

5. Association libanaise des Sciences de l'éducation.

6. Association pour le développement des méthodologies d'évaluation en éducation.

7. Association libanaise pour l'avancement des sciences.

\section{INDEX}

Mots-clés : enseignement supérieur, université

Index géographique : Liban

Keywords : higher education, universities

Palabras claves : enseñanza superior, universidad

\section{AUTEURS}

\section{YVETTE GHARIB}

Yvette Gharib est professeure associée maître de conférences, formatrice en sciences de l'éducation (Tempus, Dirasati et ADMEE), coordinatrice du cycle doctoral et chef de la commission francophone doctorale à la faculté des sciences de l'éducation de l'Université Saint Joseph (USJ) (Liban). En 2016, elle a publié (avec Patricia Rached) : « Impact de l'accompagnement intégral sur la motivation des étudiants au supérieur ", Recherche et Formation $\mathrm{n}^{\circ} 77$; (avec Sonia Constantin) « Engagement civique des étudiants : valeur essentielle pour l'USJ » (LAES). Parmi ses communications en 2016 : The Civic Role of Arab Universities (Beyrouth) ; «Effet des pratiques évaluatives des enseignements sur le sentiment d'efficacité personnelle des enseignants » (Portugal). Courriel : yvette.gharib@usj.edu.lb

\section{NISRINE HAMDAN SAADÉ}

Nisrine Hamdan Saadé est doctorante en sciences économiques, titulaire d'un master en sciences de l'éducation de l'Université Saint Joseph (Liban) et d'un DEA en sciences de l'information (Université Marne-la-Vallée). Elle est chargée d'enseignement et mène des recherches à l'Université Saint Joseph (USJ), faculté des sciences économiques (Liban), où elle coordonne également le comité pédagogique. Elle a publié en 2016 : «Une envie d'apprendre dès la première séance ", Cahiers Pédagogiques, $\mathrm{n}^{\circ} 531$ et Prêt étudiant et rendement académique : cas des étudiants de la faculté des sciences économiques (mémoire master recherche, faculté des sciences de l'éducation, USJ).

Courriel : nisrine.hamdanesaade@usj.edu.lb 